and some cooking - exemplify aspects of functional precautionary ritual behavior. This minor caveat aside, B\&L have powerfully illuminated underlying commonalities in ritual behavior.

\section{Ritual: Meaningful or meaningless?}

\section{DOI: $10.1017 /$ S0140525X0600954X}

\section{Robert Turner}

Wellcome Department of Imaging Neuroscience, Institute of Neurology, University College London, London WC1 N 3BG, United Kingdom. r.turner@fil.ion.ucl.ac.uk http://www.fil.ion.ucl.ac.uk/Turner/

Abstract: In conflating opposing meanings of the term "ritual," arising from historical Western cultural conflicts regarding church and state, this target article begs fundamental questions. Its appeals to cognitive science concepts such as "working memory" are poorly informed and obfuscate what could have been a far more penetrating and less biased discussion of stereotyped human action.

In English, it is not unusual for the same word to come to possess two almost opposite meanings - for example, the word "sanction" - which require careful distinction. The term "ritual" is similar, denoting pointless actions and also those with great meaning for participants. Boyer \& Lienard (B\&L) (and Cosmides and Tooby, whom they acknowledge as inspirations for this study) seem to have been foxed by this etymological quirk. Otherwise, their use of the term "ritual" to refer simultaneously to both opposite meanings might be regarded as disingenuous.

Much ethnography has been devoted to teasing out authentic interpretations of the rituals that are found universally in every culture (even those of university academics). Cultural anthropologists' definitions of the term "ritual" are indeed vague, for excellent reasons. Because the meanings of rituals are generally deeply embedded in the local network of social institutions and collective representations, which are to a large extent taken for granted by participants in a given culture, it is often difficult to find simple interpretations for specific component actions of any given ritual. For instance, Sperber (1975) found it quite impossible to understand why members of the tribe he studied applied butter to their hair. But absence of evidence is not evidence of absence, as all good scientists are aware.

$B \& L$ have adopted their compatriot Sperber's rationalistic bias and are content, at least at the outset of their article, to fully equate cultural ritual with the pathological and apparently irrational behaviour of humans suffering from obsessive-compulsive disorder (OCD), and with the repetitive actions of small children (who perhaps delight in their freshly-acquired ability to give order to their personal space and time). But they do not appear to appreciate that their definition of "ritualized behavior" as "stereotypy, rigidity, repetition and apparent lack of rational motivation" (target article, sect. 1), applies precisely as well to more approved, adult, and non-pathological cultural forms such as theatre, music, and poetry. It is not at all clear how these latter forms might relate to "inferred threats to fitness" arising from a "Hazard-Precaution System" (as the authors call it, perhaps using the term "system" to distance themselves from Pinker's wholesale misuse of the term "module"; see, e.g., Pinker 1997). In any case, cultural ritual clearly serves many purposes, such as worship, dedication, marking a social commitment, enacting a rite of passage, which it would be ridiculous to associate with inferred threats to fitness. Ritual is often effective in these contexts because of its dramatic power, bringing together in a choreographed and synergistic process symbols that have great resonance in the cultural understanding of its protagonists (cf. Victor Turner 1969). Perhaps B\&L have had no personal experience of this power, which would explain how easily they have confused the two opposite senses of the term "ritual."

It is also important to stress that religious ritual, like other performative genres, is rarely rigidly repetitive. On the contrary, it is often tailored to suit the occasion and/or the individuals concerned, particularly in rituals that are intended to be curative. Ritual experts frequently draw from an extended repertoire of approved variants, as do Western medical practitioners (e.g., E. L. B. Turner 1992). The relatively invariant form of the proceedings can easily be seen to provide an acceptable context or frame (see Goffman 1974) for social actions, enabling the participants to interpret them appropriately.

Far from "swamping working memory," repetitive ritual actions are typically easily memorized, and thus rapidly become overlearned, relieving any potential burden on working memory and allowing a greater focus on the affective and cognitive content of the ritual context. The reader should be aware that "swamping working memory," a favourite phrase of Boyer, is not an accepted cognitive science concept - perhaps the authors mean "increased attentional load" (e.g., Lavie 2006). Over-learning is also a vital aspect of musical performance. Indeed, humans very often rely on over-learned behaviours, consciously or unconsciously fitting themselves into predictable and thus interpretable roles. Ritual action is thus a particularly striking example of role-play.

To argue that such adherence to custom results from a narrowly defined brain Hazard-Precaution System is question-begging and has limited explanatory power, like Molière's virtus dormitiva. In my view, this aspect of human social behaviour is supported by a more "domain-general" brain system (or systems!) for planning, scenario development, and prediction, which uses Bayesian computational algorithms to imaginatively assess the potential benefits and costs of a range of possible actions. Cognitive scientists are familiar with this system as the "central executive" (Baddeley 1990; Norman \& Shallice 1980). It is obvious that the ability to acquire, learn, and represent stereotypical patterns for action greatly increases the efficiency of such neural computations by limiting the range of possibilities, and could thus increase evolutionary fitness. B\&L are tendentious in separating out actions which they happen to believe are "pointless," as the products of a special evolutionary module which could give rise to the pathological behaviour of patients with OCD.

The appeals to brain science made in this target article are also unconvincing. The authors ascribe a major role in the production of ritualized behaviour to the anterior cingulate cortex (ACC), and ascribe the symptoms of OCD to its defective performance. However, this is one of the largest anatomically defined cortical areas, and recent studies (e.g., Chein \& Schneider 2005) show that regions within it support a wide variety of functions, such as domain-general learning, emotional response, placebo effect, and internally directed attention. While indeed part of the subgenual ACC might possibly support the postulated HazardPrecaution System (Van Laere et al. 2006), this area has also been firmly implicated in mood disorder (Mayberg et al. 2005). The functional anatomy of the ACC is an area of intensive research, and the authors have reached premature conclusions.

Ultimately, B\&L reveal their disdain for what quite clearly gives most of us our major motivation, delight, and satisfaction participation in social rituals such as weddings, funerals, christenings, sporting occasions, graduations, and other initiation ceremonies - by referring to them as a "waste of time" (sect. 9.3). Such an elitist viewpoint undermines the credibility of much of this article. 